\title{
A NUMERICAL METHOD FOR KINETIC SEMICONDUCTOR EQUATIONS IN THE DRIFT DIFFUSION LIMIT
}

\author{
AXEL KLAR*
}

\begin{abstract}
An asymptotic-induced scheme for kinetic semiconductor equations with the diffusion scaling is developed. The scheme is based on the asymptotic analysis of the kinetic semiconductor equation. It works uniformly for all ranges of mean free paths. The velocity discretization is done using quadrature points equivalent to a moment expansion method. Numerical results for different physical situations are presented.
\end{abstract}

Key words. kinetic semiconductor equations, asymptotic analysis, drift-diffusion limit, numerical methods for stiff equations

AMS subject classifications. 78 A35, 82C70, 65M06

1. Introduction. Kinetic semiconductor equations are used to describe highly integrated semiconductor devices. The situation for small mean free paths is described by an asymptotic analysis. The limit equation for small mean free path of the kinetic equation with the diffusion scaling is the drift-diffusion equation.

In the drift-diffusion limit a very fine and expensive discretization depending on the mean free path has to be used for standard finite difference or particle methods due to the stiffness of the equations. This makes these schemes extremely time consuming. One way to handle the problem are domain decomposition techniques, see, e.g., [14, 16] for the semiconductor case. The basic idea is to use the computationally much cheaper limit equation where- and whenever it gives a good approximation. In all other cases the kinetic equation is used. In this approach kinetic semiconductor and macroscopic equations are solved simultaneously on different subdomains of the computational domain. A second approach is to develop directly numerical schemes for the kinetic equation working uniformly for different regimes. In particular, it should be possible to choose the discretization size independent of the mean free path. Coarse discretizations should yield a good approximation, if a near equilibrium situation prevails and the true solution is only varying slowly.

The domain decomposition approach has the advantage that well known and tested codes can be used. However, work has to be spent on the coupling procedure. In the second approach the same method can be used in the whole computational domain with possibly different sizes for the discretization. In this paper we will consider the second approach.

In recent years there has been a lot of work on numerical methods for kinetic equations in stiff regimes. For example, stationary transport equations in the diffusion limit have been considered in $[10,18,17,20]$. Nonstationary kinetic equations with a scaling leading to first order hydrodynamic equations and hyperbolic conservation laws with stiff relaxation terms are treated in $[4,5,11]$. General methods, the so called relaxation type schemes have been published in [13]. A diffusive relaxation scheme for discrete velocity models has been obtained in [12]. In the semiconductor case a moment method coupled with a semiimplicit discretization can be found in [25].

The present work considers an extension of a scheme for transport equations, see [15], which is suitable for the calculation of situations with small mean free path. The

${ }^{*}$ FB Mathematik, University of Kaiserslautern, 67663 Kaiserslautern, Germany, (klar@mathematik. uni-kl.de). 
different space time scalings involved in the problem are treated in a way proposed in [15] by using a fractional step method and a semiimplicit discretization. The time discretization can be choosen in this way independent of the mean free path. Here new problems arise due to the presence of the electric field. The main problem is to include it into the approach and to obtain a suitable discretization of the limit equation. Moreover, a new velocity discretization is introduced: Usually moment methods are widely used for semiconductor simulations, see $[6,9,22,24,25]$. We propose in this paper a scheme which is not directly based on a moment method. Instead we use a velocity discretization based on a quadrature method which is essentially equivalent to a moment method. In particular, the consideration of boundary conditions is more straightforward for this approach. Including the results of a boundary layer analysis in the scheme, kinetic boundary layers are also treated in a correct way. This allows to use coarse spatial grids even in the presence of boundary layers.

Section 2 contains a description of the results of the standard asymptotic procedure. Sections 3 and 4 contain the details of the velocity and time discretization. In Section 5 the drift-diffusion limit of the scheme is considered. In Section 6 the space discretization is presented. Section 7 contains numerical results for several examples.

2. The Equations. We consider a domain $\Omega$ in $\mathbb{R}^{3}$ and the lyinear semiconductor Boltzmann equation with parabolic band approximation

$$
\partial_{t} f+\frac{1}{\epsilon}\left(v \cdot \nabla_{x} f+\nabla_{x} \Phi \cdot \nabla_{v} f\right)+\frac{1}{\epsilon^{2}} Q(f)=G,
$$

where $f=f(x, v, t)$ is the distribution function, $x \in \Omega, v \in \mathbb{R}^{3}, t \geq 0$ and $\epsilon$ is the mean free path. $\Phi(x, t)$ denotes the electric potential,

$$
Q(f)(v)=\lambda(v) f(v)-M(v) K(f)(v)
$$

is the collision operator with weight function $M$ defined by

$$
M(v)=\frac{1}{(\pi)^{\frac{3}{2}}} \exp \left(-|v|^{2}\right)
$$

and

$$
K(f)(v)=\int s(v, w) f(w) d w
$$

where $s$ is rotationally invariant and fulfills

$$
s(v, w)=s(w, v) \geq s_{0}>0 .
$$

Moreover

$$
\lambda(v)=K(M)(v)
$$

with $\lambda(v) \leq \lambda_{0}$, where $\lambda_{0}$ is a constant. The collision operator is bounded and nonnegative on the Hilbert space $H=\mathcal{L}^{2}\left(\mathbb{R}^{3}, M^{-1}(v) d v\right)$, see [23, 25]. It has a one-dimensional kernel spanned by $M$. Moreover, the following coercivity estimate holds:

$$
<Q f, f>\geq \sigma_{0}|f-P f|^{2}
$$


with a positive constant $\sigma_{0}$. Here, we did use the notation

$$
P f(v)=\int f(w) d w M(v)
$$

for the projection onto the kernel of $Q$. Moreover, $\langle\cdot, \cdot\rangle$ and $|\cdot|$ denote the scalar product and norm in $H$. See $[23,25]$ for further details. $G=G(x, v)$ denotes the source term. The initial and boundary conditions are

$$
f(x, v, 0)=g(x, v), x \in \Omega
$$

and

$$
f(x, v, t)=k(x, v, t), x \in \partial \Omega, v \cdot n(x)<0,
$$

where $n(x)$ is the outer normal at $\partial \Omega$ at the point $x$.

Let $h=\left(h_{1}, h_{2}, h_{3}\right)$ be the solution of

$$
Q\left(h_{i} M\right)=v_{i} M, \int h_{i}(v) M d v=0
$$

for $i \in\{1,2,3\}$. Since by assumption $s$ is rotationally invariant, it follows that $\forall i, j \in\{1,2,3\}$ :

$$
\int v_{i} h_{j} M(v) d v=D \delta_{i, j}
$$

In the small mean free path limit $f(x, v, t)$ is approximated by $\rho(x, t) M(v)$, where $\rho(x, t)$ fulfills the drift-diffusion equation

$$
\partial_{t} \rho-D \nabla_{x} \cdot\left(\nabla_{x} \rho-2 \rho \nabla_{x} \Phi\right)=\tilde{G},
$$

with $\tilde{G}(x)=\int G(x, v) d v$, see [23]. Doing a boundary layer analysis, one observes that the correct zeroth order boundary conditions for the drift-diffusion equation are given by a kinetic half space problem: Let $\chi^{x}(y, v, t)$ be the bounded solution of the following halfspace problem at $x \in \partial \Omega$

$$
\begin{array}{r}
v \cdot n(x) \partial_{y} \chi^{x}+Q\left(\chi^{x}\right)=0, y \in \mathbb{R}^{-} \\
\chi^{x}(0, v, t)=k(x, v, t), x \in \partial \Omega, v \cdot n<0 .
\end{array}
$$

Then

$$
\rho(x, t) M(v)=\chi^{x}(-\infty, v, t), x \in \partial \Omega .
$$

Here $\chi^{x}(-\infty, v, t) M^{-1}(v)$ is independent of $v$.

We mention that the following scheme can be adapted to other situations, like semiconductor Boltzmann equations, where the $\delta$-distributions in energy appearing in the usual semiconductor scattering cross section are integrated out using the total energy as a new variable, see, e.g., $[6,9,22]$. In this case an integration over the unit sphere in $\mathbb{R}^{3}$ instead of the whole space $\mathbb{R}^{3}$ has to be considered. 
3. The Numerical Scheme: Velocity Discretization. The discretization of the velocity space $\mathbb{R}^{3}$ is done using a quadrature method based on Hermite polynomials. The quadrature method is essentially equivalent to a moment method for the semiconductor equation. The same approach substituting the expansion in Hermite polynomials by an expansion in Legendre polynomials can be done for the above mentioned kinetic semiconductor equation with an integration over the unit sphere. This is a well known way of proceeding in transport theory, see [19]. For semiconductor equations usually the moment equations are treated directly, see [25] for an approach treating the moment system associated to equation (2.1) numerically and an investigation of the convergence of moment methods.

It is assumed that the distribution function can be expanded in Hermite polynomials:

$$
f(v)=\psi(v) M(v)
$$

with

$$
\psi(v)=\sum_{k, l, m=0}^{K, L, M} \psi_{k, l, m} N_{k, l, m} H_{k, l, m}(v)
$$

where $H_{k, l, m}(v)=H_{k}\left(v_{1}\right) H_{l}\left(v_{2}\right) H_{m}\left(v_{3}\right)$ and $N_{k, l, m}=N_{k} N_{l} N_{m} . H_{k}$ is the one dimensional $k$-th order Hermite polynomial and $N_{k}^{2}=\frac{1}{2^{k} k !}$ is the normalization factor. $\psi_{k, l, m}$ is given by

$$
\psi_{k, l, m}=\int \psi(v) H_{k, l, m}(v) N_{k, l, m} M(v) d v .
$$

The moment equations obtained with this expansion are essentially equivalent to a velocity discretization based on a quadrature method where the zeros of the Hermite polynomials and the related weights for Hermite integration [1] are used:

Writing $v^{i}=\left(v_{1}^{i_{1}}, v_{2}^{i_{2}}, v_{3}^{i_{3}}\right), i=\left(i_{1}, i_{2}, i_{3}\right), i_{1}=0, \cdots, K, i_{2}=0, \cdots, L, i_{3}=0, \cdots, M$, where $v_{1}^{i_{1}}, v_{2}^{i_{2}}, v_{3}^{i_{3}}$ are the zeros of $H_{K+1}, H_{L+1}, H_{M+1}$, and $z^{i}$ for the associated weights one obtains

$$
\psi_{k, l, m}=\int \psi(v) H_{k, l, m}(v) N_{k, l, m} M(v) d v=\sum_{j} \psi^{j} H_{k, l, m}^{j} N_{k, l, m} M^{j} z^{j}
$$

with $M^{j}=M\left(v^{j}\right), \psi^{j}=\psi\left(v^{j}\right)$ and $H_{k, l, m}^{j}=H_{k, l, m}\left(v^{j}\right)$. The last equality is exact due to the properties of Hermite integration, since the degree of the polynomial $\psi H_{k, l, m}$ is small enough. For the collision operator we get, using first (3.1) and then (3.2) and defining

$$
s_{k, l, m}^{i}=\int H_{k, l, m}(w) M(w) s\left(v^{i}, w\right) d w
$$

the following

$$
\begin{aligned}
& K(\psi M)\left(v^{i}\right)=\int s\left(v^{i}, w\right) \psi(w) M(w) d w \\
= & \sum_{k, l, m=0}^{K, L, M} N_{k, l, m} \psi_{k, l, m} \int H_{k, l, m}(w) M(w) s\left(v^{i}, w\right) d w
\end{aligned}
$$




$$
\begin{aligned}
& =\sum_{k, l, m=0}^{K, L, M} N_{k, l, m} \psi_{k, l, m} s_{k, l, m}^{i} \\
& =\sum_{j} \psi^{j} s_{i, j}
\end{aligned}
$$

with

$$
s_{i, j}=\sum_{k, l, m=0}^{K, L, M} s_{k, l, m}^{i} N_{k, l, m}^{2} H_{k, l, m}^{j} z^{j} M^{j} .
$$

This is exact, if the coefficients $s_{k, l, m}^{i}$ are evaluated exactly. The derivative with respect to the velocity variable is approximated by

$$
\begin{array}{r}
\partial_{v_{1}} \psi=\partial_{v_{1}}\left(\sum_{k, l, m=0}^{K, L, M} \psi_{k, l, m} H_{k, l, m} N_{k, l, m}\right) \\
=\sum_{k=1}^{K} \sum_{l, m=0}^{L, M} \psi_{k, l, m} 2 k H_{k-1} H_{l} H_{m} N_{k, l, m}
\end{array}
$$

due to (3.1) and since the Hermite polynomials fulfill $H_{k}^{\prime}=2 k H_{k-1}$. Therefore, using (3.2) we have

$$
\partial_{v_{1}} \psi\left(v^{i}\right)=\sum_{j} \psi^{j} c_{i, j}^{1}
$$

with

$$
c_{i, j}^{1}=\sum_{k=1}^{K} \sum_{l, m=0}^{L, M} 2 k H_{k-1, l, m}^{i} N_{k, l, m}^{2} H_{k, l, m}^{j} M^{j} z^{j}
$$

$\partial_{v_{2}}, \partial_{v_{3}}$ are treated in the same way yielding the coefficients $c_{i, j}^{2}, c_{i, j}^{3}$. Using

$$
\nabla_{v}(\psi M)=M \nabla_{v} \psi-2 \psi v M
$$

this yields the velocity discretized version of equation (2.1) at the discretization point $v^{i}$ :

$\partial_{t} \psi^{i}+\frac{1}{\epsilon}\left(v^{i} \nabla_{x} \psi^{i}+\nabla_{x} \Phi \cdot\left(\nabla_{v} \psi\right)^{i}-2 v^{i} \cdot \nabla_{x} \Phi \psi^{i}\right)+\frac{1}{\epsilon^{2}}\left(\lambda^{i} \psi^{i}-(K(\psi M))^{i}\right)=\tilde{G}^{i}$

with

$$
\begin{array}{r}
\left(\nabla_{v} \psi\right)^{i}=\left(\sum_{j} \psi^{j} c_{i, j}^{1}, \sum_{j} \psi^{j} c_{i, j}^{2}, \sum_{j} \psi^{j} c_{i, j}^{3}\right) \\
(K(\psi M))^{i}=\sum_{j} \psi^{j} s_{i, j} \\
\lambda^{i}=\lambda\left(v^{i}\right) .
\end{array}
$$

In terms of the distribution function $f$ we have

$$
\partial_{t} f^{i}+\frac{1}{\epsilon}\left(v^{i} \nabla_{x} f^{i}+\nabla_{x} \Phi \cdot\left(\nabla_{v} f\right)^{i}\right)+\frac{1}{\epsilon^{2}}(Q(f))^{i}=G^{i} .
$$


with

$$
\begin{array}{r}
f^{i}=f\left(v^{i}\right) \\
\left(\nabla_{v} f\right)^{i}=\left(\nabla_{v} \psi\right)^{i} M^{i}-2 v^{i} \psi^{i} M^{i} \\
(Q(f))^{i}=\left(\lambda^{i} \psi^{i}-(K(\psi M))^{i}\right) M^{i} .
\end{array}
$$

The matrices $c_{i, j}^{n}, n=1,2,3$ and $s_{i, j}$ are computed in advance using the recursion properties of the Hermite polynomials.

4. Time Discretization. In this section we develop a time discretization that is able to treat varying mean free paths $\epsilon$ with a fixed discretization. It is not necessary to adapt the time step once the mean free path tends to 0. Moreover, the scheme is in the limit $\epsilon \rightarrow 0$ a good discretization of the drift diffusion equation.

We proceed similiar as in [15] and use the asymptotic procedure and a fractional step scheme with a semiimplicit discretization. The aim of the procedure is to treat exactly those terms for which it is necessary in an implicit way. One writes $f$ as $f=f_{0}+\epsilon f_{1}$ and collects suitable terms together, such that only terms on the scale $\frac{1}{\epsilon^{2}}$ are involved:

Let $\left(f_{0}, f_{1}\right)$ be the solution of the set of equations

$$
\begin{aligned}
\partial_{t} f_{0}+v \cdot \nabla_{x} f_{1}+\nabla_{x} \cdot \Phi \nabla_{v} f_{1} & =-\frac{1}{\epsilon^{2}} Q\left(f_{0}\right)+G(x) \\
\partial_{t} f_{1} & =\frac{1}{\epsilon^{2}}\left(-v \cdot \nabla_{x} f_{0}-\nabla_{x} \Phi \cdot \nabla_{v} f_{0}-Q\left(f_{1}\right)\right) .
\end{aligned}
$$

We take inital and boundary values

$$
f_{0}(x, v, 0)+\epsilon f_{1}(x, v, 0)=g(x, v), x \in \Omega, v \in \mathbb{R}^{3}
$$

and

$$
f_{0}(x, v, t)+\epsilon f_{1}(x, v, t)=k(x, v, t), x \in \partial \Omega, v \cdot n<0 .
$$

The second boundary condition for the system (4.1) is

$$
f_{0}(x, v, t)-\epsilon f_{1}(x, v, t)=q(x, v, t), x \in \partial \Omega, v \cdot n>0
$$

with $q$ arbitrary. One observes that $f_{0}+\epsilon f_{1}$ fulfills the original equation (2.1) and the initial and boundary conditions. It is therefore the desired solution of the original problem.

The results of the boundary layer analysis, see, e.g. $[3,23]$, are included in the scheme by choosing $q$ in the following way:

Let $\chi^{x}(y, v, t)$ be the solution of the halfspace problem (2.4). Since the outgoing function at the boundary for the kinetic problem (2.4) for $\epsilon$ tending to 0 is the same as the outgoing solution of the half space problem, we define

$$
q(x, v, t)=\chi^{x}(0, v, t), x \in \partial \Omega, v \cdot n>0 .
$$

It is obviously not reasonable to determine the outgoing function by solving the halfspace problem. This would need too much computing time. Here a fast approximate scheme as in [7] or [14] is needed. For example, a first approximation is given by choosing simply an approximation $\tilde{\chi}^{x}(-\infty, v, t)$ of the asymptotic value $\chi^{x}(-\infty, v, t)$ of the halfspace problem as the outgoing function:

$$
q(x, v, t)=\tilde{\chi}^{x}(-\infty, v, t), x \in \partial \Omega, v \cdot n>0 .
$$


The simplest approximation of $\chi^{x}(-\infty, v, t)$ is given by equalizing the half range fluxes of the halfspace problem at 0 and $\infty$ :

$$
\tilde{\chi}^{x}(-\infty, v, t)=\frac{\int_{w \cdot n<0} w \cdot n k(x, w, t) d w}{\int_{w \cdot n<0} w \cdot n M(w) d w} M(v)=\bar{k}(x, v, t) .
$$

A more sophisticated approximation for $q$, see [14], is given by

$$
\begin{aligned}
& \tilde{\chi}^{x}(-\infty, v, t)=\bar{k}(x, v, t)+ \\
& \frac{1}{D} \int_{w \cdot n<0}(w \cdot n)^{2}[k(x, w, t)-\bar{k}(x, w, t)] d w M(v)
\end{aligned}
$$

and

$$
\begin{aligned}
q(x, v, t)= & \tilde{\chi}^{x}(-\infty, v, t) \\
+ & M(v) \int_{w \cdot n<0} \frac{w \cdot n}{(w-v) \cdot n}\left[s(v, w)-\frac{(w \cdot n)^{2}}{D}\right] \\
& \quad\left[k(x, w, t)-\tilde{\chi}^{x}(-\infty, v, t)\right] d w
\end{aligned}
$$

for $x \in \partial \Omega, v \cdot n>0$. We remark that a correct treatment of the boundary conditions is important, in particular, if zeroth order kinetic boundary layers are present and one is using a coarse spatial grid not resolving the layer. Using $(4.3,4.4)$ or $(4.5,4.6)$ one obtains a good approximation of the solution with a first order boundary layer even if only a very coarse grid is used. See Figure 7.4 in Section 7 or reference [15] for some examples. Higher order approximations of the half space problem may be useful in certain situations as well.

The system of equations (4.1) will be solved with a fractional step scheme:

Step 1:

$$
\begin{aligned}
\partial_{t} f_{0}+v \cdot \nabla_{x} f_{1}+\nabla_{x} \Phi \cdot \nabla_{v} f_{1} & =G(x) \\
\partial_{t} f_{1} & =0
\end{aligned}
$$

Step 2:

$$
\begin{aligned}
& \partial_{t} f_{0}=-\frac{1}{\epsilon^{2}} Q\left(f_{0}\right) \\
& \partial_{t} f_{1}=\frac{1}{\epsilon^{2}}\left(-v \cdot \nabla_{x} f_{0}-\nabla_{x} \Phi \cdot \nabla_{v} f_{0}-Q\left(f_{1}\right)\right)
\end{aligned}
$$

For Step 1 an explicit discretization will be used, Step 2 is discretized semi-implicitely to treat the stiffness of the equations in a correct way.

Let $\Delta t$ denote the time step and $f_{0}^{k}, f_{1}^{k}, k=0, \cdots, n=\frac{t}{\Delta t}$ the approximations of $f_{0}(x, v, k \Delta t), f_{1}(x, v, k \Delta t)$. The initial and boundary values are given as above. The time discretization is then given by the following:

Step 1:

$$
\begin{array}{r}
f_{0}^{k+\frac{1}{2}}=f_{0}^{k}-\Delta t\left(v \cdot \nabla_{x} f_{1}^{k}+\nabla_{x} \Phi \cdot \nabla_{v} f_{1}^{k}\right)+G \Delta t \\
f_{1}^{k+\frac{1}{2}}=f_{1}^{k}
\end{array}
$$


Step 2:

$$
\begin{aligned}
& f_{0}^{k+1}=f_{0}^{k+\frac{1}{2}}+\frac{\Delta t}{\epsilon^{2}}\left(-Q\left(f_{0}^{k+1}\right)-P f_{0}^{k+1}+P f_{0}^{k+\frac{1}{2}}\right) \\
& f_{1}^{k+1}=f_{1}^{k+\frac{1}{2}}+ \\
& \frac{\Delta t}{\epsilon^{2}}\left[-v \cdot \nabla_{x} f_{0}^{k+1}-\nabla_{x} \Phi \cdot \nabla_{v} f_{0}^{k+1}-Q\left(f_{1}^{k+1}\right)-P f_{1}^{k+1}+P f_{1}^{k+\frac{1}{2}}\right]
\end{aligned}
$$

Rewriting (4.8) we obtain

$$
f_{0}^{k+1}+\frac{\Delta t}{\epsilon^{2}}\left(Q\left(f_{0}^{k+1}\right)+P f_{0}^{k+1}\right)=f_{0}^{k+\frac{1}{2}}+\frac{\Delta t}{\epsilon^{2}} P f_{0}^{k+\frac{1}{2}}
$$

and

$$
\begin{aligned}
& f_{1}^{k+1}+\frac{\Delta t}{\epsilon^{2}}\left(Q\left(f_{1}^{k+1}\right)+P f_{1}^{k+1}\right) \\
= & f_{1}^{k+\frac{1}{2}}+\frac{\Delta t}{\epsilon^{2}} P f_{1}^{k+\frac{1}{2}}-\frac{\Delta t}{\epsilon^{2}}\left(v \cdot \nabla_{x} f_{0}^{k+1}+\nabla_{x} \Phi \cdot \nabla_{v} f_{0}^{k+1}\right) .
\end{aligned}
$$

This leads to

Step 2:

$$
\begin{aligned}
f_{0}^{k+1} & =A f_{0}^{k+\frac{1}{2}}+B P f_{0}^{k+\frac{1}{2}} \\
f_{1}^{k+1} & =A f_{1}^{k+\frac{1}{2}}+B\left[P f_{1}^{k+\frac{1}{2}}-v \cdot \nabla_{x} f_{0}^{k+1}-\nabla_{x} \Phi \cdot \nabla_{v} f_{0}^{k+1}\right]
\end{aligned}
$$

where the operator $A$ is defined by

$$
A=\left(I+\frac{\Delta t}{\epsilon^{2}}(Q+P)\right)^{-1}=\frac{\epsilon^{2}}{\Delta t}\left(\frac{\epsilon^{2}}{\Delta t} I+Q+P\right)^{-1}
$$

and

$$
B=\frac{\Delta t}{\epsilon^{2}} A=\left(\frac{\epsilon^{2}}{\Delta t} I+Q+P\right)^{-1} .
$$

Here $I$ denotes the identity. The operator

$$
\left(\frac{\epsilon^{2}}{\Delta t} I+Q+P\right)
$$

is positive and invertible for all $\epsilon \geq 0, \Delta t>0$ due to the above properties of $Q$. The coercivity estimate $(2.2)$ gives

$$
\begin{aligned}
& \left|\left(\frac{\epsilon^{2}}{\Delta t} I+Q+P\right) g\right||g| \geq<\left(\frac{\epsilon^{2}}{\Delta t} I+Q+P\right) g, g> \\
\geq & \frac{\epsilon^{2}}{\Delta t}|g|^{2}+\sigma_{0}|g-P g|^{2}+|P g|^{2} \geq\left(\frac{\epsilon^{2}}{\Delta t}+\sigma\right)|g|^{2} .
\end{aligned}
$$

with $\sigma=\min \left(1, \sigma_{0}\right)$. This gives

$$
\left|\left(\frac{\epsilon^{2}}{\Delta t} I+Q+P\right)^{-1}\right| \leq \frac{1}{\sigma+\frac{\epsilon^{2}}{\Delta t}} .
$$


For example in the case of a relaxation model with $M K=P$ and $\lambda=1$ we obtain

$$
A=\left(1+\frac{\Delta t}{\epsilon^{2}}\right)^{-1} I
$$

and

$$
B=\left(\frac{\epsilon^{2}}{\Delta t}+1\right)^{-1} I
$$

In this case the semi-implicit scheme has a very simple formulation. In general, in each time step we have to solve in Step 2 two linear Fredholm integral equations of the form

$$
\left(\frac{\epsilon^{2}}{\Delta t} I+Q+P\right) f=g
$$

This may be achieved by standard methods $[2,8]$. If the number of velocity discretization points is not too large, i.e. the degree of the expansion into Hermite polynomials is not too high, one can invert the matrix derived from the velocity discretization of $\frac{\epsilon^{2}}{\Delta t} I+Q+P$ in advance with a direct method. The resulting matrix is stored for the computation. This gives a very efficient method to handle the Fredholm equation.

5. The Small Mean Free Path Limit. In this section we investigate the behaviour of the time discretized scheme as $\epsilon$ tends to 0 for fixed $\Delta t$.

As $\epsilon \rightarrow 0$ the operators $A$ and $B$ have the following behaviour: We have for $\Delta t$ fixed and $\epsilon$ small due to (4.10)

$$
|A|=\frac{\epsilon^{2}}{\Delta t}\left|\left(\frac{\epsilon^{2}}{\Delta t} I+Q+P\right)^{-1}\right| \leq \frac{\epsilon^{2}}{\Delta t} \frac{1}{\sigma+\frac{\epsilon^{2}}{\Delta t}}=O\left(\frac{\epsilon^{2}}{\Delta t}\right)
$$

and

$$
\begin{aligned}
& \left|B-(Q+P)^{-1}\right|=\frac{\epsilon^{2}}{\Delta t}\left|\left(\frac{\epsilon^{2}}{\Delta t} I+Q+P\right)^{-1}(Q+P)^{-1}\right| \\
\leq & \frac{\epsilon^{2}}{\Delta t} \frac{1}{\sigma\left(\sigma+\frac{\epsilon^{2}}{\Delta t}\right)}=O\left(\frac{\epsilon^{2}}{\Delta t}\right) .
\end{aligned}
$$

Using these estimates we get that the scheme reduces in the diffusion limit, $\epsilon$ tending to 0 , to the following

Step 1:

$$
\begin{array}{r}
f_{0}^{k+\frac{1}{2}}=f_{0}^{k}-\Delta t\left(v \cdot \nabla_{x} f_{1}^{k}+\nabla_{x} \Phi \cdot \nabla_{v} f_{1}^{k}\right)+G \Delta t \\
f_{1}^{k+\frac{1}{2}}=f_{1}^{k}
\end{array}
$$

Step 2:

$$
\begin{aligned}
f_{0}^{k+1} & =(Q+P)^{-1} P f_{0}^{k+\frac{1}{2}} \\
f_{1}^{k+1} & =(Q+P)^{-1}\left[P f_{1}^{k+\frac{1}{2}}-v \cdot \nabla_{x} f_{0}^{k+1}-\nabla_{x} \Phi \cdot \nabla_{v} f_{0}^{k+1}\right]
\end{aligned}
$$

Moreover, we have

$$
(Q+P) P f=P f
$$




$$
(Q+P)(h(v) M(v))=v M(v)
$$

with $h$ defined in Section 2,

$$
\nabla_{v} f_{0}^{k+1}=\nabla_{v}\left(P f_{0}^{k+1}\right)=-2 v P f_{0}^{k+1}
$$

due to the definintion of $P$ and

$$
P\left(\nabla_{v} f_{1}^{k}\right)=\int \nabla_{v} f_{1}^{k} d v M(v)=0
$$

This yields

Step 2:

$$
\begin{aligned}
f_{0}^{k+1} & =P f_{0}^{k+\frac{1}{2}} \\
f_{1}^{k+1} & =P f_{1}^{k+\frac{1}{2}}-h(v) \cdot\left(\nabla_{x}\left(P f_{0}^{k+1}\right)-\nabla_{x} \Phi 2 P f_{0}^{k+1}\right)
\end{aligned}
$$

Considering Step 2 and Step 1 together we obtain for $\epsilon=0$

$$
\begin{aligned}
& f_{0}^{k+1}=P f_{0}^{k+1}=P f_{0}^{k+\frac{1}{2}} \\
= & P f_{0}^{k}-\Delta t\left(\nabla_{x} \cdot P\left(v f_{1}^{k}\right)+\nabla_{x} \Phi \cdot P\left(\nabla_{v} f_{1}^{k}\right)\right)+G \Delta t .
\end{aligned}
$$

Using (5.1) and

$$
P\left(v f_{1}^{k}\right)=-D\left(\nabla_{x} P f_{0}^{k}-2 \nabla_{x} \Phi P f_{0}^{k}\right)
$$

one obtains

$$
P f_{0}^{k+1}=P f_{0}^{k}+\Delta t D \nabla_{x} \cdot\left(\nabla_{x} P f_{0}^{k}-2 \nabla_{x} \Phi P f_{0}^{k}\right)+G \Delta t
$$

or

$$
\Theta^{k+1}=\Theta^{k}+\Delta t D \nabla_{x} \cdot\left(\nabla_{x} \Theta^{k}-2 \nabla_{x} \Phi \Theta^{k}\right)+\tilde{G} \Delta t .
$$

This is an explicit time discretization for the drift-diffusion equation. The boundary conditions for the drift-diffusion equation that are given in the limit by the solution of the halfspace problem (2.4) fit to the boundary conditions for the kinetic scheme as defined in the last section.

6. Space Discretization. We restrict from now on for notational simplicity to the case, where $f_{0}$ and $f_{1}$ depend only on the first space coordinate: The domain under consideration is $[0, L]$.

We define a staggered grid $x_{i}=i \Delta x, i=0, \cdots, N$ with $N=\frac{L}{\Delta x}$, and $x_{i-\frac{1}{2}}=$ $\left(i-\frac{1}{2}\right) \Delta x, i=0, \cdots, N+1$.

We use the notation

$$
\begin{gathered}
f_{0}^{k}=f_{0}^{k}(i) \sim f_{0}(i \Delta x, v, k \Delta t), \\
f_{1}^{k}=f_{1}^{k}(i) \sim f_{1}\left(\left(i-\frac{1}{2}\right) \Delta x, v, k \Delta t\right)
\end{gathered}
$$

and

$$
\Phi=\Phi(i) \sim \Phi(i \Delta x) .
$$


The discretization of the initial values is straightforward. The boundary conditions are discretized by

$$
\begin{aligned}
& f_{0}^{k}(0)+\frac{\epsilon}{2}\left(f_{1}^{k}(0)+f_{1}^{k}(1)\right)=k(0, v, t), v_{1}>0 \\
& f_{0}^{k}(0)-\frac{\epsilon}{2}\left(f_{1}^{k}(0)+f_{1}^{k}(1)\right)=q(0, v, t), v_{1}<0
\end{aligned}
$$

and

$$
\begin{gathered}
f_{0}^{k}(N)+\frac{\epsilon}{2}\left(f_{1}^{k}(N)+f_{1}^{k}(N+1)\right)=k(L, v, t), v_{1}<0 \\
f_{0}^{k}(N)-\frac{\epsilon}{2}\left(f_{1}^{k}(N)+f_{1}^{k}(N+1)\right)=q(L, v, t), v_{1}>0 .
\end{gathered}
$$

Let the operators $D_{+}$and $D_{-}$be defined as

$$
\begin{aligned}
& D_{+} f(i)=f(i+1)-f(i) \\
& D_{-} f(i)=f(i)-f(i-1)
\end{aligned}
$$

and $S_{+}^{\Phi}, S_{-}^{\Phi}$ by

$$
\begin{aligned}
& S_{+}^{\Phi} f(i)=B(\Phi(i+1)-\Phi(i)) f(i+1)-B(\Phi(i-1)-\Phi(i)) f(i) \\
& S_{-}^{\Phi} f(i)=B(\Phi(i)-\Phi(i-1)) f(i)-B(\Phi(i-1)-\Phi(i)) f(i-1)
\end{aligned}
$$

with

$$
B(x)=\frac{1}{2}\left(1-\frac{x}{e^{x}-1}\right) .
$$

We mention that for $x$ small $B(x) \sim \frac{x}{2}$. Discretizing in (4.7) and (4.9) $\partial_{x} f_{0}$ with $D_{-}, \partial_{x} f_{1}$ with $D_{+}$and approximating $\nabla_{x} \Phi \cdot \nabla_{v} f_{0}$ by $\frac{S_{-}^{\Phi}}{\Delta x}\left(\nabla_{v} f_{0}\right)$ and $\nabla_{x} \Phi \cdot \nabla_{v} f_{1}$ by $\frac{S_{+}^{\Phi}}{\Delta x}\left(\nabla_{v} f_{1}\right)$ yields the following scheme

Step 1:

$$
\begin{array}{r}
f_{0}^{k+\frac{1}{2}}=f_{0}^{k}-\Delta t\left(v_{1} \frac{D_{+}}{\Delta x} f_{1}^{k}+\frac{S_{+}^{\Phi}}{\Delta x}\left(\nabla_{v} f_{1}^{k}\right)\right)+G \Delta t \\
f_{1}^{k+\frac{1}{2}}=f_{1}^{k}
\end{array}
$$

Step 2:

$$
\begin{aligned}
f_{0}^{k+1} & =A f_{0}^{k+\frac{1}{2}}+B P f_{0}^{k+\frac{1}{2}} \\
f_{1}^{k+1} & =A f_{1}^{k+\frac{1}{2}}+B\left[P f_{1}^{k+\frac{1}{2}}-v_{1} \frac{D_{-}}{\Delta x} f_{0}^{k+1}-\frac{S_{-}^{\Phi}}{\Delta x}\left(\nabla_{v} f_{0}^{k+1}\right)\right]
\end{aligned}
$$

In the limit for small $\epsilon$ we obtain the space discretized diffusion equation

$$
\begin{aligned}
f_{0}^{k+1}= & P f_{0}^{k+1}=P f_{0}^{k+\frac{1}{2}}=P f_{0}^{k}-\Delta t \frac{D_{+}}{\Delta x} P\left(v_{1} f_{1}^{k}\right)+G \Delta t \\
& =P f_{0}^{k}+\frac{\Delta t}{(\Delta x)^{2}} D D_{+}\left(D_{-} P f_{0}^{k}-2 S_{-}^{\Phi} P f_{0}^{k}\right)+G \Delta t
\end{aligned}
$$


or

$$
\Theta^{k+1}=\Theta^{k}+\frac{\Delta t}{(\Delta x)^{2}} D D_{+}\left(D_{-} \Theta^{k}-2 S_{-}^{\Phi} \Theta^{k}\right)+\tilde{G} \Delta t .
$$

This can be written as

$$
\Theta^{k+1}=\Theta^{k}+\frac{\Delta t}{(\Delta x)^{2}} D D_{+} \tilde{S}_{-}^{\Phi} \Theta^{k}+\tilde{G} \Delta t
$$

where $\tilde{S}_{-}^{\Phi}$ is defined analogously to $S_{-}^{\Phi}$ with

$$
\tilde{B}(x)=\frac{x}{e^{x}-1}
$$

instead of $B$. This is an explicit Scharfetter-Gummel type discretization, see [21], of the drift diffusion equation. In particular, we obtain independent of the size of the discretization $\Delta x$ a good discretization of the limit equation for all ranges of the mean free path. We observe, that, assuming $\tilde{G}=0$, we need in the limit a relation like

$$
\Delta t \leq \frac{(\Delta x)^{2}}{2 D} \frac{1}{\tilde{B}\left(-\Delta x E_{\max }\right)}
$$

with

$$
E_{\text {max }}=\sup _{x}\left|\frac{\partial \Phi}{\partial x}(x)\right|
$$

as for the explicitely differenced drift-diffusion equation, to obtain positivity and stability of our scheme. For small electric fields this reduces to the standard restriction on the time step for the diffusion equation

$$
\Delta t \leq \frac{(\Delta x)^{2}}{2 D}
$$

For $\Delta x$ small (6.3) is approximately

$$
\Delta t \leq \frac{(\Delta x)^{2}}{2 D}\left(1-E_{\max } \frac{\Delta x}{2}\right)
$$

Condition (6.3) may be relaxed for $\epsilon$ large to a standard CFL-condition for the transport equation.

7. Numerical Results and Examples. In this section a numerical study of the scheme is presented. We restrict to slab geometry, i.e. $x \in[0, L], L=1$ and consider $M K=P, \lambda=1$, i.e. a relaxation time approximation. The solutions are computed with the semi-implicit scheme derived above for different space and velocity discretizations.

The velocity discretization is done using Hermite discretization points as explained in section 3 . For comparison a uniform velocity discretization is implemented as well, see Figure 7.1 and 7.2.

To obtain positivity and stability of the semi-implicit scheme in the limit $\epsilon$ tending to 0 one has to take - for a fixed space discretization $\Delta x$ - a time step $\Delta t$ of the size given by (6.3). In particular, this means that the size of $\Delta t$ can be chosen independent of $\epsilon$. In general, the CPU time needed for one time step for the semiimplicit scheme is 
about two times as large as the CPU time needed for one time step of a straightforward explicit discretization of (2.1). Since the time step for the explicit discretization has to be chosen of the order $\epsilon^{2}$ this yields a considerable gain of computation time in situations with small mean free path compared to the fully explicit discretization. For $\epsilon$ small the relation of the CPU time for the semiimplicit and the fully explicit discretization is in the present case approximately given by

$$
2 \epsilon^{2}: \frac{(\Delta x)^{2}}{2} \frac{1}{\tilde{B}\left(-\Delta x E_{\max }\right)}
$$

if the desired accuracy does not require a smaller time step than the one that can be used for the semiimplicit scheme. This means, that - at least for not too large $\Delta x$ and $E_{\text {max }}$ - one obtains an advantage in CPU time if the number of mean free paths per cell is larger than approximately 2. For a detailed comparison of the scheme for transport equations with explicit and fully implicit schemes see [15]. In particular, the size of the time step to achieve a certain accuracy has been determined there for different situations and the CPU time has been compared in more detail.

The solution of the kinetic equation computed by the scheme derived above is in the following computed for different physical situations. The figures show that in case the physical situation allows coarse discretizations - the scheme gives a good resolution of the true solution, if such discretizations are used. In particular, in the presence of boundary layers coarse discretization not resolving the layer may be used without loosing accuracy in the bulk of the domain.

Example 1: In this example the electric potential is choosen to be 0 except in a potential well located in the left half of the slab. The maximal electric field is 10. $G$ is equal to 0 . The initial condition is $M(v)$ except in the right half of the slab, where the density is increased in the beginning. The boundary conditions are $f(0, v)=M(v), v_{1}>0, f(L, v)=M(v), v_{1}<0$. This is a nonequilibrium solution due to the large Knudsen number. The boundary conditions are equilibrium conditions.

Example 2: We consider the same situation as in Example 1, but with boundary conditions $f(0, v, t)=v_{1} M(v), v_{1}>0$. The solution of this problem has a kinetic boundary layer at $x=0$. This is an equilibrium situation in the bulk of the domain and a nonequilibrium situation at the boundary.

Example 3: As Example 2, but the electric field is constant equal to 1. The initial condition is constant equal to $M$. The boundary conditions are $f(0, v, t)=$ $v_{1} M(v), v_{1}>0, f(L, v, t)=0, v_{1}<0$. The solution of this problem has a kinetic boundary layer at $x=0$ as in Example 2 .

The solutions are plotted using different space discretization with $\Delta x$ ranging from 0.005 to 0.1 and a velocity discretization with a number of quadrature points ranging from 2 to 16 for the semi-implicit scheme. The time discretization is chosen due to the stability condition (6.3) for $\epsilon$ small. For large $\epsilon$ the restriction on the time step is relaxed to a CFL-type condition for the kinetic equation (2.1).

Example 1 is used to investigate the influence of the order of the velocity discretization. A large number of space discretization points, $\Delta x=0.005$, is used. The reference solution is the solution with a very fine discretization. The solution of the drift diffusion equation is computed by the limit explicit scheme (5.3). We plot in Figure $7.1(\epsilon=1.0)$ and Figure $7.2(\epsilon=0.1)$ the solutions computed by the semiimplicit scheme with Hermite quadrature with different numbers of quadrature points and the solution computed by a scheme with uniform velocity discretization with 8 discretization points. Here, one observes a clear advantage of the discretization de- 


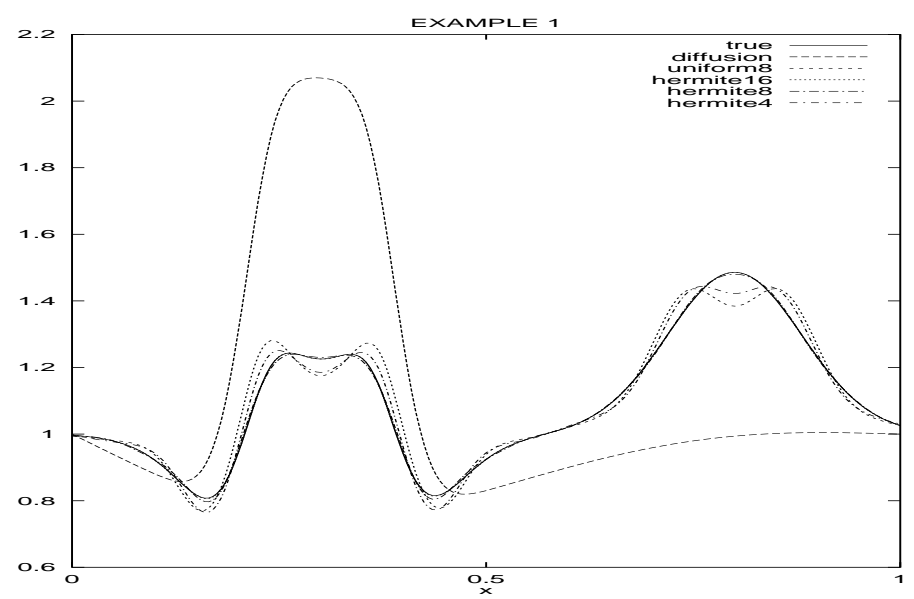

FIG. 7.1. Density for $\epsilon=1.0, t=0.1$.

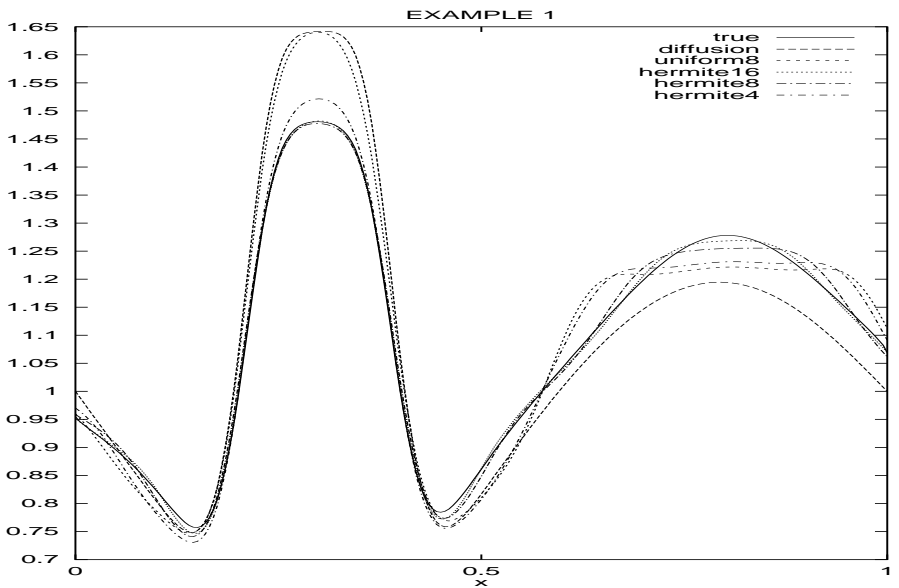

FIG. 7.2. Density for $\epsilon=0.1, t=0.03$.

rived by the moment expansion method. 8 or 16 quadrature points already yield a reasonable approximation. In this example no $\mathrm{CPU}$ time is gained compared to the explicit scheme due to the large Knudsen numbers.

In Figure 7.3 the situation in Example 2 is considered with $\epsilon=0.001$. For the space discretization we use $\Delta x=0.01$, i.e. we have 10 mean free paths per cell. Here one observes that a lower degree of expansion into Hermite polynomials is sufficient to obtain good accuracy, at least outside of the kinetic layer. A discretization with only 4 quadrature points leads already to good results outside the kinetic layer. We plot the reference solution and the solution of the drift-diffusion equation with boundary coefficients derived from the halfspace problem. The boundary values for the semiimplicit scheme are found by determining approximately the outgoing distribution of the halfspace problem (2.4) using $(4.5,4.6)$. In this example the explicit scheme with the same space discretizatioin needs about 25 times more CPU time than the present scheme. Moreover, the present scheme is as accurate as the explicit one.

Example 3 is used to study the influence of the space discretization. $\epsilon$ is again 


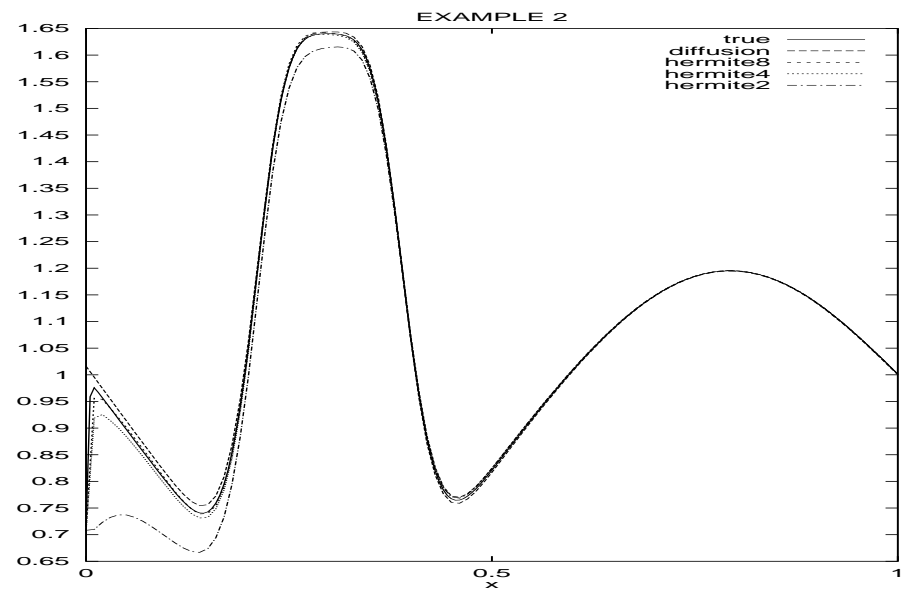

FIG. 7.3. Density for $\epsilon=0.001, t=0.03$.

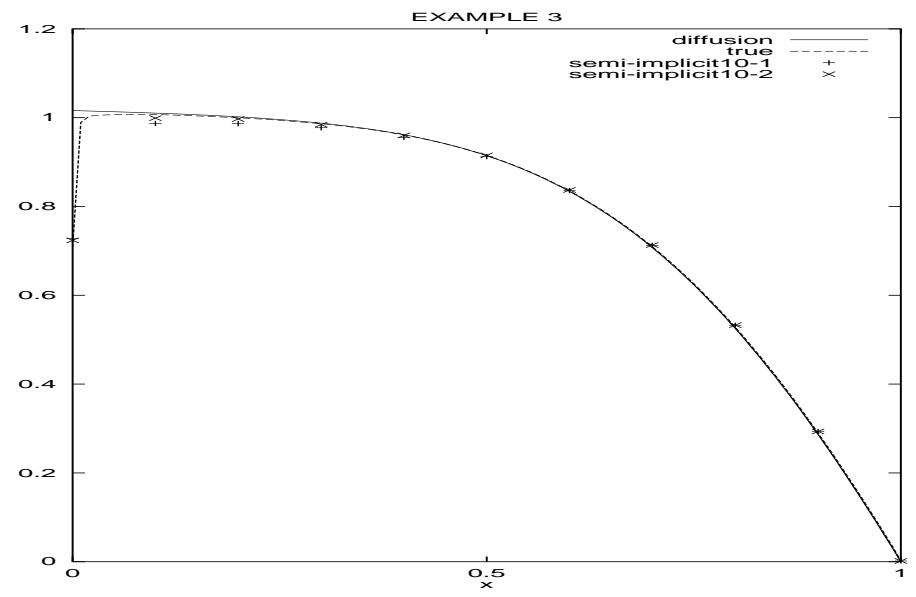

FIG. 7.4. Density for $\epsilon=0.001, t=0.1$.

0.001 . The number of velocity points that is used is 16 . Since the electric field and the initial distribution are uniform in the whole domain a fine space discretization is not necessary. We use only 10 spatial cells in the whole domain. The boundary values for the semiimplicit scheme are found by determining approximately the outgoing distribution of the halfspace problem (2.4) using the two methods described in section 3 , i.e. $(4.3,4.4)$ and $(4.5,4.6)$. The results are denoted in Figure 7.4 by 'semi-implicit101 ' and 'semi-implicit10-2', respectively. One observes in Figure 7.4 that even for a coarse diffusive discretization (100 mean free paths per cell) the solution is found with very good accuracy despite the fact that the kinetic boundary layer is not resolved. Moreover, one observes that the accuracy is improved using formula (4.6). We mention that other approaches to obtain the correct discrete boundary conditions using coarse space discretizations for stationary transport equations can be found in $[10,17]$. The scheme developed here is in this example about 250 times faster as the explicit scheme with the same space discretization. 
8. Conclusions. The scheme presented in this work has the following properties:

- A natural velocity discretization is given by a Hermite quadrature corresponding to a moment expansion into Hermite polynomials.

- The semiimplicit time discretization allows to take a fixed time step independent of the order of the mean free path.

- The limiting scheme for small mean free paths is an explicit ScharfetterGummel type discretization of the drift diffusion equation.

- Boundary layers are included in the scheme by solving approximately a half space problem.

- The scheme allows to take coarse velocity, time and space discretizations, if the physical situations is allowing them. This means it is suitable for the computation of limit situations.

\section{Acknowledgements:}

I am grateful to Jan Mohring for helpful discussions.

\section{REFERENCES}

[1] M. Abramowitz And I. Stegun, Handbook of mathematical functions, Dover Publications, New York, 1972.

[2] K. AtKinson, A Survey of Numerical Methods for the solution of Fredholm Integral Equations of the Second Kind, Society for industrial and applied mathematics, Philadelphia, 1976.

[3] A. Bensoussan, J. Lions, and G. Papanicolaou, Boundary layers and homogenization of transport processes, Publ. RIMS Kyoto Univ, 15 (1979), p. 52.

[4] I. Bobylev AND J. Struckmeier, Implicit and iterative methods for the Boltzmann equation, TTSP, 25 (1996), p. 175.

[5] R. CAflisch, S. Jin, AND G. Russo, Uniformly accurate schemes for hyperbolic systems with relaxation, to appear in SIAM J. Num. Anal.

[6] A. Gnudi, D. Ventura, F. Baccarani, And F. Odeh, Two dimensional MOSFET simulation by means of multidimensional spherical harmonics expansion of the Boltzmann transport equation, Sol. State Electron., 36 (1993), p. 575.

[7] F. GOLSE AND A. KLAR, A numerical method for computing asymptotic states and outgoing distributions for kinetic linear half space problems, J. Stat. Phys., 80 (1995), p. 1033.

[8] W. HACKBUSCH, Integralgleichungen: Theorie und Numerik, LAMM 68, Teubner, 1989, 1989.

[9] K. HennaCy And N. Goldsman, A generalized Legendre polynomial/sparse matrix approach for determining the distribution function in non-polar semiconductors, Sol. State Electron., 38 (1995), p. 1485.

[10] S. Jin AND D. LeVERMORE, Fully-discrete numerical transfer in diffusive regimes, TTSP, 22 (1993), p. 739.

[11] - Numerical schemes for hyperbolic conservation laws with stiff relaxation terms, J. Comp. Phys., 126 (1996), p. 449.

[12] S. Jin, L. PARESChI, AND G. TOSCANI, Diffusive relaxation schemes for discrete-velocity kinetic equations, SIAM J. Num. Anal., (to appear).

[13] S. JIN AND Z. XIN, The relaxation schemes for systems of conservation laws in arbitrary space dimensions, Comm. Pure Appl. Math., 48 (1995), p. 235.

[14] A. KLAR, Asymptotic-induced domain decomposition methods for kinetic and drift diffusion semiconductor equations, to appear in SIAM J. Sci. Comp.

[15] — An asymptotic-induced scheme for nonstationary transport equations in the diffusive limit, to appear in SIAM J. Num. Anal.

[16] H. Kosina, Simulation des Ladungstransportes in elektronischen Bauelementen mit Hilfe der Monte Carlo Methode, Dissertation, TU Wien, 1992.

[17] E. LARSEn AND J. MOREL, Asymptotic solution of numerical transport problems in optically thick, diffusive regimes II, J. Comp. Phys., 83 (1989), p. 212.

[18] E. Larsen, J. Morel, AND W. Miller, Asymptotic solution of numerical transport problems in optically thick, diffusive regimes, J. Comp. Phys., 69 (1987), p. 283.

[19] E. Lewis And W. Miller, Computational Methods of Neutron Transport, John Wiley and Sons, 1984.

[20] T. Manteuffel, S. McCormick, J. Morel, S. Oliveira, and G. Yang, A fast multigrid algorithm for isotropic transport problems I: Pure scattering, SIAM J. Sci. Comp., 16 
(1995), p. 601.

[21] P. Markowich, The Stationary Semiconductor Device Equations, Springer, 1986.

[22] J. Mohring, The Spherical Harmonics Method, Dissertation, Univ. of Kaiserslautern, 1996.

[23] F. Poupaud, Diffusion approximation of the linear semiconductor equation, J. Asympt. Anal., 4 (1991), p. 293.

[24] C. RINGHOFER, An expansion method for the Boltzmann transport equation using an adaptive Galerkin procedure, preprint, Arizona State University, (1995).

[25] C. SChMeISER AND A. ZwIrChmayr, Convergence of moment methods for the semiconductor Boltzmann equation, preprint, TU Wien, (1996). 